\title{
Trivium
}

Revue franco-allemande de sciences humaines et sociales - Deutsch-französische Zeitschrift für Geistesund Sozialwissenschaften

$10 \mid 2012$

Lisibilité

\section{Qu'est-ce donc qu'une trace, et quelle est sa fonction épistémologique ? État des lieux}

\section{Sybille Krämer}

Traducteur : Cécile Chamayou-Kuhn

\section{CpenEdition}

Journals

Édition électronique

URL : http://journals.openedition.org/trivium/4171

ISSN : 1963-1820

\section{Éditeur}

Les éditions de la Maison des sciences de l'Homme

\section{Référence électronique}

Sybille Krämer, «Qu'est-ce donc qu'une trace, et quelle est sa fonction épistémologique ? État des

lieux », Trivium [En ligne], 10 | 2012, mis en ligne le 30 mars 2012, consulté le 07 septembre 2020. URL : http://journals.openedition.org/trivium/4171

Ce document a été généré automatiquement le 7 septembre 2020.

\section{(c) (1) $\odot$}

Les contenus des la revue Trivium sont mis à disposition selon les termes de la Licence Creative Commons Attribution - Pas d'Utilisation Commerciale - Pas de Modification 4.0 International. 


\title{
Qu'est-ce donc qu'une trace, et quelle est sa fonction épistémologique? État des lieux
}

\author{
Sybille Krämer \\ Traduction : Cécile Chamayou-Kuhn
}

\section{NOTE DE L'ÉDITEUR}

Nous remercions Sybille Krämer ainsi que la maison d'édition Suhrkamp de nous avoir accordé l'autorisation de traduire ce texte pour le présent numéro.

\section{Des questions à foison}

1 Est-il possible que la lecture de traces ne soit pas seulement le reste archaïque d'une "connaissance sauvage», les balbutiements de la métaphysique, le stade d'une herméneutique sans texte ? Est-il possible que cette lecture ne soit pas seulement une forme première et instinctive de grammaires symboliques, mais qu'elle se rencontre dans toutes les pratiques où entrent en jeu les signes, la connaissance et l'interprétation? La lecture des traces est-elle une pratique du savoir qui ne s'emploie pas seulement dans les sciences humaines, mais aussi dans les sciences naturelles, et qui s'avère par ailleurs être un exemple de la manière dont les pratiques quotidiennes et les processus scientifiques se rejoignent?

2 Mais de quelle manière la lecture de traces peut-elle être différenciée de la lecture d'un texte ? Comment cette lecture se démarque-t-elle de l'interprétation de signes textuels et iconographiques?

3 Peut-on ranger les traces dans la catégorie des signes, des indices ou des symptômes, ou existe-t-il quelque chose qui différencie les cendres, c'est-à-dire la trace d'un feu, de la fumée, c'est-à-dire le signe d'un feu? Est-il possible de ne plus réduire la lecture de 
traces à un support qui aide à accéder à des formes "supérieures" de l'emploi de signes et de symboles et (inversement), est-il possible d'obtenir ce qui constitue un signe sur le modèle de la trace? Les signes conventionnels et les traces laissées involontairement forment-ils deux catégories d'objets différentes ou ceux-ci n'ouvrentils pas plutôt deux perspectives distinctes, au travers desquelles chaque phénomène sémiotique doit être considéré?

Les traces doivent-elles leur existence à « l'écriture aveugle de la causalité ", autrement dit, ont-elles été laissées de manière aléatoire et sans intention aucune, ou bien existet-il - dans le contexte d'expériences scientifiques ou de performances artistiques par exemple - des «traces mises en scène » ? Découvre-t-on les traces ou celles-ci naissentelles lors de l'acte de lecture ? Les conventions et les usages culturels entrent-ils en jeu dans l'acte de lecture des traces? Les traces sont-elles donc des faits sociaux, tel un indice judiciaire qui doit être reconnu comme une preuve, ou font-elles partie du monde muet des choses?

5 Autant de questions qui circonscrivent un champ dans lequel ce volume ${ }^{1}$ souhaite s'inscrire pour y apporter certaines réponses.

\section{Pourquoi ouvrir maintenant un débat sur la trace et la lecture des traces?}

6 La conception traditionnelle de la représentation qu'exprime la formule aliquid stat pro aliquo a acquis, à l'époque moderne, une nouvelle signification : le postulat de Saussure, selon lequel la valeur d'un signe naît uniquement dans sa relation aux autres signes ${ }^{2}$, et l'approche de Cassirer, qui soutient qu'il nous faut concevoir la réalité comme une variété des formes symboliques par lesquelles tout ce qui nous est donné devient une construction interprétative ${ }^{3}$, conduisent à la conclusion suivante: il n'existe (plus) aucun accès au monde et au réel qui ne soit exempt de signes et qui ne dépende d'une interprétation. Voilà, tout au moins, l'une des idées centrales de l'époque moderne.

7 Il est frappant de constater que le développement technique contemporain fonctionne de la même manière : la digitalisation suggère que tout ce qui possède un corps peut être transformé en une structure de données, dans la mesure où l'alphabet binaire s'avère être «l'atome » d'un langage universel dans lequel ce qui nous est donné peut être « inscrit » ou - comme c'est le cas pour les informations génétiques - est inscrit depuis toujours. Aussi le double constat concernant, d'une part, l'absence de référentialité des signes et, d'autre part, l'irréfutable textualité du monde qui règne dans la pensée "postmoderne", en apparait-il comme la conséquence directe: dématérialisation, déréalisation, décorporalisation, informatisation, virtualisation, euphorie de la simulation - ce ne sont là que différentes expressions caractérisant une tendance qui consiste à libérer les signes des liens qu'ils entretiennent avec ce qui n'en a précisément pas les caractéristiques. Par là même, cette tendance consiste à poser le signe en tant que nature du monde comme un absolu. Pourtant, c'est ainsi que les choses disparaissent.

8 Or, est-il possible que la réflexion ouverte autour du concept de trace soit justement fructueuse et contemporaine parce qu'elle permet d'opposer à la fluctuation des signes, que l'on qualifiera d'insouciante et d'aréférentielle, des éléments qui trouvent leur ancrage dans une sorte de "sémantique des choses ${ }^{4}$ ? De fait, les actes de 
« représenter " , de " lire », d'« interpréter » acquièrent dans le contexte de la lecture des traces une signification qui ne s'épuise pas dans les systèmes de signes à eux seuls. Dans le cadre d'une réflexion sur la trace, nous allons poursuivre l'étude du discours sur la sémiologie et la représentation, tout en maintenant dans nos mains le fil d'Ariane qui nous conduit hors du " pur » monde des signes et qui nous relie aux choses, à la corporéité et la matérialité du monde, laquelle est la condition sine qua non de la genèse ainsi que de l'intelligibilité des traces. Les traces sont-elles donc le point nodal à partir duquel le sens naît sur la base de l'absence de sens ? Mais, qu'est-ce qu'une trace ?

\section{La signification du mot « trace»}

Commençons par l'étymologie. "Spur» [la «trace » en Allemand, NdT], en latin "vestigium », vient du vieux haut-allemand «spor » et signifie à l'origine l'empreinte de pied. Selon l'histoire du terme, "Spüren » (« ressentir »), i. e. l'acte qui consiste à relever et à suivre une piste, est intimement lié à cette signification ${ }^{7}$. L'objet (la trace) et l'activité (ressentir) entrent en relation de manière élémentaire, toutefois non parce que cette activité se rapporte à l'acte de produire des traces - comme cela paraitrait évident, mais plutôt à l'acte de les interpréter et de les suivre. Ce n'est donc pas la formation d'une trace, mais plutôt l'emploi qui en est fait après sa genèse qui est à l'évidence la forme d'activité "correspondant » à la trace. Est-il donc ainsi établi que seule l'utilisation qui est faite d'une trace la constitue comme telle?

" usuel de «trace », en tant que "suite d'empreintes ou d'impressions laissées sur le sol par le passage de quelqu'un ou de quelque chose $»^{8}$ n'est pas le seul à renvoyer à la scène primitive de l'empreinte de pied. Les acceptions techniques de ce terme renvoient elles aussi au fait de "suivre une piste » : lorsque "Spur» ("piste ») fait référence à la partie d'une bande magnétique, lorsqu'une "Busspur » ("voie de bus ») est réservée aux autobus et fermée aux automobilistes, lorsque des trains électriques possèdent différents «Spurweiten » (écartements de voies) ou qu'une voiture « aus der Spur brechen " (quitte la voie en freinant)- dans toutes ces situations, "Spur» (la trace) désigne le respect d'une direction précise et bien définie. «In der Spur bleiben » ("rester sur la voie») signifie donc suivre un cours spécifique, à la manière dont le train suit des rails ou un téléphérique suit un câble porteur. De l'acte consistant à chercher une piste, nous sommes ainsi arrivés à une opération réglementée, voire, pour reprendre l'exemple de l'écartement des voies des trains, à une norme !

11 On mentionnera ensuite l'une des dernières acceptions de ce mot: très tôt déjà, on utilisait Spur pour parler d'une quantité infime, d'une bricole, dans le sens de ce qui n'est repérable et perceptible qu'à l'état de "Spurenelement » (oligoélément), voire de ce qui fait défaut : on dit par exemple qu'il manque « eine Spur Salz " (une pincée de sel) dans la soupe, ou qu'il n'y a « keine Spur didaktischen Geschicks » (pas une once de didactique) chez tel enseignant, ou encore qu'il ne pèse pas l'ombre d'un soupçon sur le voisin [« keine Spur von Verdacht »]. Est donc inscrit au cœur de la notion de trace non seulement tout ce qui est nettement perceptible, i. e. les normes qui doivent être respectées, mais aussi ce qui est à peine perceptible, ce qui se situe à la limite de l'imperceptible.

12 Pour autant, il convient de se concentrer, dans les propos suivants, sur l'acception élémentaire du mot «Spur », dont l'étymologie nous rappelle qu'elle signifie la trace de 
pas ou la piste. Aussi nous faut-il à présent étudier plus avant les caractéristiques qui fondent notre conception de la trace.

\section{Les caractéristiques de la trace}

\section{L'absence}

Dans le creux que laisse apparaitre une empreinte, et par lequel un mouvement dans le temps prend forme dans l'espace, on peut voir que quelqu'un ou quelque chose est passé. La présence de la trace témoigne de l'absence de ce qui l'a formée. Dans la visibilité de la trace, ce qui l'a engendrée se dérobe à nous et demeure invisible : la piste permet certes d'émettre des déductions, mais cette reconstruction ne sera jamais qu'une reproduction, une représentation dont le caractère imagé indique toujours l'absence de ce qui est représenté. Une empreinte laissée par un contact physique ne permet aucunement d'identifier avec certitude ce qui l'a laissée ${ }^{9}$. La trace ne rend jamais présent ce qui est absent ; elle représente la non-présence de l'Absent. Les traces ne donnent pas à voir ce qui est absent, mais plutôt l'absence même. « Le trait tracé sur le sable n'est pas l'élément d'un sentier, mais le vide même de la passée ${ }^{10}$. Est-ce donc à dire que la lecture de traces constitue une sorte de «métaphysique du quotidien »?

\section{La performance de l'orientation}

14 Il s'agit toujours pour ceux qui lisent les traces de déterminer une orientation valable pour leur propre conduite, qu'elle soit pratique ou théorique. La lecture de traces devient nécessaire à la condition que naissent l'incertitude, l'insécurité, voire la peur et, en somme, à condition que survienne une situation qui ne nous est pas familière (ou ne l'est plus ${ }^{11}$. Un problème - qu'il soit de nature pratique ou théorique - apparaît lorsque commence la lecture des traces. Les lecteurs de traces sont en effet mus par des intérêts particuliers et ils poursuivent des objectifs spécifiques. L'attention, nécessaire lors de la lecture de traces qui sont toujours imperceptibles dans un premier temps, est par conséquent toujours une «attention ciblée ».

\section{La matérialité}

Les traces se présentent physiquement à nos yeux ; sans signature physique, il n'y a pas de trace. Les traces sont engendrées par un contact, c'est-à-dire qu'elles naissent matériellement : elles sont perceptibles dans et sur un matériau donné. Les traces font partie du monde des choses. Ce n'est que parce qu'il existe une continuité dans la matérialité, la corporéité et la sensorialité du monde qu'il est possible de laisser ou de lire des traces ${ }^{12}$. Le lien entre l'auteur d'une trace et la trace doit être pensé selon les termes d'une relation de cause à effet; il ne repose ni sur une similitude (comme c'est le cas dans la représentation) ni sur une convention (comme pour le symbole). Contrairement au signe, la matérialité de la trace n'est pas subordonnée à la représentation. Les traces ne représentent pas; elles présentent quelque chose. Par ailleurs, comme tout objet, elles ne font que désigner quelque chose et ne parlent pas. 


\section{Le dérangement} l'inconnu surgit en terrain connu, ou lorsque ce que l'on attend n'arrive pas. Ce n'est qu'à travers les déviations que les traces deviennent perceptibles. "La trace authentique [...] dérange l'ordre du monde ${ }^{13}$.» En outre, une forme de puissance, la force de s'inscrire et de s'imprimer, de laisser ses marques sur quelque chose, doit être propre à ce qui se manifeste dans la trace. Les traces n'apparaissent que dans la mesure où une forme existante est effacée puis reconfigurée sous l'effet d'une réécriture. Quiconque est étranger à l'espace dans lequel il se meut laisse des traces. Les traces correspondent à l'irruption d'un au-delà inconnu au sein d'un ici-bas familier.

\section{L'arbitraire}

On ne fabrique pas une trace, on la laisse, et ce sans intention aucune. De même, effacer des traces revient à en laisser une. Et vice versa: dès lors qu'une trace est sciemment laissée et mise en scène en tant que telle, il ne s'agit plus d'une trace. Seul ce qui n'est pas intentionnel, ce qui est involontaire, incontrôlé, arbitraire, grave ou dessine ces lignes de rupture qui peuvent être lues comme des pistes. À la différence du signe que nous créons, la signification d'une trace existe au-delà de l'intention de celui qui la génère. C'est justement ce qui échappe à notre attention, à notre contrôle ou à notre vigilance qui, à partir de nos actes, prend la forme d'une trace: ce n'est pas la conscience mais le « poids » et la matérialité de l'Être qui forgent des traces.

\section{La dépendance d'un observateur et d'une action}

Une chose n'est pas une trace, pourtant elle est lue comme telle. Pour que de "simples » choses deviennent des traces, il importe que l'on soit animé par des intérêts ciblés et guidé par une perception sélective de l'environnement. Nous sommes impliqués dans la lecture des traces ${ }^{14}$. Par ailleurs, les traces se forment sous les yeux de celui qui les observe. Une trace n'est que ce qui est considéré et tenu pour tel. Cela fait-il pour autant des traces des faits sociaux dont l'essence repose sur la reconnaissance qu'ils appellent?

19 Le caractère involontaire propre à la formation de traces correspond, en tout état de cause, à la volonté du lecteur des traces. L'inattention de celui qui laisse des traces et l'attention de celui qui les lit, qui les trouve et les identifie, constituent les deux facettes de la trace. Toutefois, il existe des situations dans lesquelles il importe précisément de ne pas percevoir des traces manifestes.

\section{Interprétation, narration et polysémie}

Bien que les traces soient dues à la "contrainte aveugle » de deux corps exerçant un effet l'un sur l'autre, on ne les trouve pas; on les fait naître en les interprétant. Lire une trace signifie intégrer l'ordre perturbé, qui a permis la formation d'une trace, à un nouvel ordre, et le transposer dans cet ordre ; cela se produit lorsque l'événement qui a donné lieu à une trace est reconstruit à la manière d'une narration. La sémantique de la trace ne se déploie qu'au sein d'une « logique » de la narration, dans laquelle la trace se 
dote de son propre "lieu narré ". Pourtant, il existe toujours une multitude de narrations de ce genre. C'est la raison pour laquelle les traces sont polysémiques : cette caractéristique est constitutive de la trace, en ce sens il est impossible d'en faire abstraction. Une chose qui ne peut que faire l'objet d'une seule interprétation et ne peut avoir qu'une signification n'est pas une trace, mais plutôt le signe de quelque chose.

\section{Rupture temporelle}

21 La trace donne à voir une chose qui est définitivement révolue au moment de sa lecture. L'« essence » de la trace est en effet « d'avoir été ». Par conséquent, les traces peuvent se décomposer et se désintégrer. À la différence de l'indice, qui renvoie toujours à des choses qui arrivent en même temps sans être pour autant visibles en même temps, il existe toujours un décalage temporel entre l'acte consistant à laisser une trace et celui consistant à la lire : cette non-simultanéité doit être conçue comme l'ordre de la trace. Deux régimes temporels se croisent dans la trace. Ceci ne vaut pas seulement pour le rapport qu'entretiennent le passé et le présent, mais aussi pour celui qu'entretiennent le présent et le futur. L'art de la divination, la mantique ${ }^{15}$, peut être interprété comme une lecture de traces inversée.

\section{Unidimensionnalité et irréversibilité}

L'asymétrie de la rupture temporelle trouve un écho dans l'unidimensionnalité de l'« événement communicationnel», dans son irréversibilité et son manque de réciprocité. Les traces sont et demeurent muettes. Dans la mesure où la trace exprime quelque chose par le biais de l'interprétation narrative, il s'agit d'une communication totalement unidimensionnelle, dans laquelle toute interchangeabilité des rôles joués par l'« émetteur » et le « récepteur » demeure exclue.

\section{Médialité, hétéronomie, passivité}

Les traces sont hétéronomes. Elles partagent cette ingérence, ainsi que cette extériorité radicale, avec tous les médias qui endossent pour nous le rôle de «messagers». Un messager parle toujours d'une voix étrangère. La détermination provenant d'un ordre extérieur, qui apparaît sous cet angle, met en exergue une structure de la passivité. Celle-ci est constitutive de la trace. Cela vaut grossièrement pour la réceptivité d'un matériau qui doit être "souple», c'est-à-dire dont la structure interne doit être suffisamment molle pour permettre la formation d'une trace: une trace de pas ne s'imprime pas sur du sable gelé16. La passivité de la trace se manifeste plus distinctement encore, puisque celle-ci n'est pas automatique, mais puisque elle naît (et s'efface) sous l'effet d'« une activité extérieure ». Et ce, non seulement parce qu'une trace est laissée inconsciemment, mais parce que seule la personne qui déchiffre une trace la forme à partir de choses ou d'inscriptions.

\section{La lecture des traces}

Le lien étymologique existant entre le mot «trace » et "l'acte de suivre une trace » a déjà attiré notre attention sur le fait qu'« être une trace » et celui d'« être lu comme 
une trace " présentent certaines similitudes. Toutefois, si les traces sont (seulement) générées par l'acte de lecture, alors l'acte dont il s'agit ici fait partie de la préhistoire de la lecture de textes: de fait, avant même que le terme "lire" signifie la forme de perception et de transformation de l'écriture, il veut simplement dire ramasser, sélectionner, ordonner et préparer. La «sélection de traces» est un processus fastidieux et complexe qui ne saurait se contenter de trouver son objet sur place pour le lire, mais qui doit prioritairement le constituer par la sélection entre ce qui peut (probablement) être interprété comme une trace dans un champ de perception et ce qui n'est (probablement) pas une trace ${ }^{17}$. Identifier une trace signifie que l'on peut opérer une distinction entre ce qui est une trace et ce qui n'en est pas une ${ }^{18}$. La fécondité de cette distinction correspond à une transformation: les altérations et les troubles intervenant dans l'ordre familier des choses se transforment en une image cohérente, celle qui résulte d'une trace narrative ${ }^{19}$. Les traces doivent s'assembler, à la manière de fragments, pour donner une forme pleine. Plus les traces seront nombreuses, plus cela sera réussi. Il est troublant de voir sur la plage la trace isolée d'un pied droit à côté de laquelle manquent, soit le pied gauche, soit la trace du pas qui le précède ou le suit. L'ordre généré lors de la lecture de traces peut être qualifié de « réseau » : souvent, ce sont plusieurs choses qui s'assemblent pour former une trace. De plus, la police scientifique, lors du relevé de traces, a souvent recours à des signes et à des images conventionnels : les empreintes digitales ne peuvent être considérées comme une trace menant au criminel que dans le contexte de données personnelles, de désignations de témoins et d'empreintes supplémentaires ${ }^{20}$.

Les traces représentent en somme le lieu où les choses muettes "se mettent à parler " grâce à notre intuition. L'« intuition » implique pourtant la chose suivante : ce n'est que parce que nous connaissons, reconnaissons et considérons la vie ainsi que les lois propres au monde matériel que nous pouvons transformer des fragments perceptibles de ce monde en autant d'interprétations. Or, celles-ci portent témoignage de ce qui nous échappe et qui demeure pour nous invisible. Lors de la lecture de traces, la matérialité se révèle être la condition de l'immatérialité et l'immanence la condition de la transcendance.

\section{6. "Le relevé de traces »: un art et une expérience esthétique}

La lecture de traces est un savoir-faire et, en ce sens, il s'agit aussi d'un art. Il n'est aucunement étonnant que la méthode du relevé de traces soit tout d'abord apparue comme technique artistique dans le contexte de l'arte povera, avant de faire l'objet d'une réflexion en tant que pratique épistémologique dans les domaines de l'histoire des sciences, des sciences de la culture et de la philosophie à la fin des années $70^{21}$. En 1974, une exposition intitulée Le relevé de traces. Archéologie et mémoire (Spurensicherung. Archäologie und Erinnerung) réunissait à Hambourg les œuvres d'une série d'artistes ayant travaillé indépendamment les uns des autres sur la collection, l'adaptation pseudo-scientifique et l'exposition, quasi-muséographique qui comprenait des objets trouvés dont les caractéristiques étaient les suivantes: ils étaient minuscules, accessoires, fortuits et appartenaient à la sphère privée. De plus, ils étaient traités par ces artistes comme les témoignages d'une exploration anthropologique de l'existence humaine ${ }^{22}$. Ces mises en scène esthétiques n'avaient pas pour objectif de faire du relevé 
de traces une méthodologie objectivable ni, pour les artistes, de s'ériger en précurseurs d'une «science des indices ». Il s'agissait plutôt de thématiser les questionnements, les lacunes interprétatives et les incertitudes qui sous-tendent toute lecture de traces, ainsi que de présenter la rupture entre deux ordres, à savoir ce qui est présent et ce qui est absent, la mémoire personnelle et le message réifié, un passé dans lequel des reliques du passé sont encore des objets d'usage courant et un présent de l'expérience esthétique intervenant " après coup ", dans lequel ces reliques ne sont plus perçues que comme des traces ${ }^{23}$. Le courant artistique nommé Spurensicherung, que l'on peut traduire par "relevé de traces", a traité le thème de la trace d'un point de vue subversif et a paradoxalement joué un rôle d'avant-garde pour la réhabilitation de la lecture des traces en tant que méthodologie scientifique. Par là même, nous abordons la lecture des traces en tant que pratique scientifique.

\section{Les « arts scientifiques » ou la lecture des traces}

La lecture des traces est un savoir-faire; il s'agit d'un "art de la supposition (intelligente) ${ }^{24}$, c'est-à-dire d'une pratique qui mène, le cas échéant, à un nouveau savoir, mais qui nous laisse découvrir, dans le même temps, les limites de ce dernier. On distingue au moins cinq traditions, ainsi que cinq champs de signification cognitive de la trace et de créativité épistémique relative à la lecture de traces ${ }^{25}$.

\section{Le flair : une technique d'orientation élémentaire}

La lecture des traces est un art scientifique du quotidien; il conduit à se confronter à des situations d'incertitude, dans lesquelles l'invisible se déduit du visible, avant de servir à orienter la conduite de nos vies. Il s'agit là de ce que l'on appelle la " connaissance sauvage", celle qui repose sur la juste vision des choses, le "flair ", l'intuition et l'expérience des chasseurs, des bergers et des nomades, des femmes savantes, des prêtres ou des guérisseurs, qui reconstruisent une réalité passée à partir de traces présentes en filigrane ou sont capables de projeter un avenir prévisible ${ }^{26}$. La lecture des traces se fait grâce à un flair presque instinctif que l'on rapprochera de celui des animaux ou des instincts qui assurent leur survie. De même, l'art de la prédiction et la mantique, c'est-à-dire l'art de l'interprétation dans des «situations où la visibilité est réduite ${ }^{27}$ sont les modalités d'un comportement qui vise à suivre des traces pour s'orienter. Le flair se sert d'expériences relatives à des événements qui se produisent communément, sans qu'il soit pour autant nécessaire de sonder les liens de causalités que ceux-ci peuvent entretenir : ainsi, le médecin diagnostique une maladie en interprétant des symptômes, une condition météorologique à venir peut être déduite de la forme d'un nuage, le commissaire retrouve les indices laissés par un criminel.

Charles Sanders Peirce a introduit le concept d'«abduction " (dérivé du latin abductio, ou "action d'enlever») comme troisième forme de conclusion possible après la déduction et l'induction. Il postule en outre qu'elle est la seule conclusion vraiment novatrice, dans la mesure où elle introduit une nouvelle idée. Les abductions sont à replacer dans le contexte de la découverte, c'est-à-dire dans la «logic of discovery ». Bien que Peirce souligne le « caractère logique » de la constitution abductive du savoir ${ }^{28}$, il admet dans le même temps que l'abduction ne fait que supposer ce qui peut avoir 
lieu $^{29}$. Ainsi l'abduction est-elle proche du fait de deviner et, au vu du jugement extrêmement rapide qu'elle implique, elle ne peut pas être justifiée ${ }^{30}$. Peirce considère (également) l'abduction comme étant le résultat de l'évolution. Il lui attribue par conséquent des fondements biologiques et souligne par ailleurs que selon lui, elle n'est pas seulement de nature cognitive, mais qu'elle est aussi accompagnée d'une émotion et d'une satisfaction physique ${ }^{31}$. Est-ce donc à dire que la lecture de traces en tant qu'art scientifique archaïque et que technique d'orientation élémentaire se rapproche de la conclusion abductive ${ }^{32}$ ?

\section{La trace du souvenir} mémoire ${ }^{33}$. Platon associait déjà fermement le thème de la formation de la mémoire à celle des traces ${ }^{34}$. Selon lui, les perceptions se gravent dans l'âme - laquelle est du reste un cadeau de Mnémosyne - à la manière des empreintes dans la cire. Le creux que laisse apparaître l'empreinte se remplit ensuite dans la mémoire parce que l'on reconnaît quelque chose, c'est-à-dire, dès lors que le contenu d'un objet extérieur correspond à la forme de l'empreinte laissée dans l'âme. Leibniz généralise cette explication engrammique de la mémoire par l'inscription de traces pour en faire le principe de toute substance et de toute représentation. Chaque monade représente le monde dans une perspective individuelle et unique. Cette perspective se forge grâce à des traces du passé, mais aussi à des traits appartenant à l'avenir ${ }^{35}$. Toutefois, à la différence de Platon, pour lequel la mémoire est liée à une capacité consciente de se souvenir, la plupart des traces demeurent indétectables pour Leibniz, car les perceptions des monades sont souvent insignifiantes, confuses et indistinctes. Ce n'est que lorsque les traces latentes deviennent une aperception et qu'elles font l'objet d'une réflexion qu'elles parviennent à la conscience, constituant ainsi le contenu manifeste du souvenir ${ }^{36}$. «La présence indélébile de l'indétectable » ne peut donc, selon Leibniz, accéder que rétrospectivement à la conscience ${ }^{37}$.

Sigmund Freud va plus loin encore dans la mesure où, pour lui, la mémoire et la conscience s'excluent l'une l'autre. Il explique la capacité d'enregistrement et l'efficacité de l'inconscient à l'aide du "bloc-notes magique ", c'est-à-dire d'une tablette à écrire sur laquelle on peut effacer les notes que l'on a prises. Les stimuli du monde extérieur viennent s'y graver, telles des traces durables comparables à des écrits dans le creux d'une mémoire demeurant inconsciente, alors que l'on peut retranscrire sans limite des expériences à la surface ${ }^{38}$.

\section{Métaphysique de la trace}

Depuis plus de 2000 ans, la trace subsume des concepts philosophiques qui détectent ou interprètent dans la finitude une référence à l'infinitude et dans le visible une référence à l'invisible. Dans ce contexte, la formation des traces a une valeur ontologique et épistémologique. Pour Plotin, le réel est aussi une trace; il constitue, en ce sens, un ensemble de références ${ }^{39}$. Entre les corps matériels, qui représentent la strate inférieure, et l'Un divin, la sphère la plus élevée, il existe une gradation dans laquelle chaque palier est mis en place par celui qui se situe au-dessus de lui, si bien qu'il en porte les traces. Ces traces représentent en retour l'ascension vers la connaissance que l'on comparera à l'Échelle de Jacob. Le monde corporel visible devient 
la trace d'un Dieu lointain. C'est l'idée centrale introduite par Plotin dans la philosophie néoplatonicienne, et que suit Saint Augustin lorsqu'il affirme que l'«unitatis vestigium $»^{40}$, la trace de l'Un, est gravée sur l'ensemble des créatures du monde sous la forme d'une spéculation de la Trinité. Plotin mentionne déjà que la trace indique la voie à la connaissance. Or, ceci est ensuite interprété par Bonaventure et plus tard par Nicolas de Cues et Giordano Bruno, non seulement comme une explication positive des possibilités de notre connaissance, mais aussi comme une exposition négative des limites imposées à la connaissance.

$\mathrm{Au}$ XXe siècle, ce sont surtout Heidegger, Lévinas et Derrida qui abordent le thème de la trace sous un angle nouveau. Plus précisément, ils l'abordent dans le cadre d'une critique et d'une déconstruction de la métaphysique: la trace devient le lieu de la différence et de l'altéritét ${ }^{41}$. Heidegger oppose à l'utilitarisme rationnel de la technique l'expérience du réel pré-théorique mise en forme par la poésie. Cette expérience, qui constitue en principe la trace de l'absence du divin, échappe au geste visant tant une identification terminologique qu'une utilisation par la technique. Cette expérience permet par ailleurs d'avoir conscience de l'indigence de notre existence ${ }^{42}$. Pour Lévinas, le concept de trace renvoie aux limites de l'interprétabilité ou de l'intelligibilité de l'Autre, qui, précisément, ne saurait être interprété. Ce n'est pas à la rationalité technologique que s'oppose Lévinas quand il met au jour la trace comme trait caractéristique de l'Autre, mais à l'accaparement sémiologico-herméneutique de l'Autre, étant entendu que cet accaparement est subordonné à l'égologie d'un sujet maître de $\operatorname{soi}^{43}$. Quant à Derrida, il met en corrélation la sémiologie et la trace, soutenant que tout signe devient la trace des autres signes par une différence qui le renvoie à la fois vers tous les autres et l'exclut en même temps de ceux-ci. Ainsi la trace constitue-t-elle le fondement de notre rapport symbolique au monde. Derrida nomme cela "différance ", désignant par ce terme une incessante dynamique de renvois et de différenciations qui nous empêche à jamais, par et dans la pensée, de rejoindre une origine ou encore une conclusion définitive ${ }^{44}$.

\section{Le paradigme de l'indice}

Dans la perspective de méthodes sémiologiques, l'indexicalité est une possibilité d'analyse propre aux sciences humaines. Carlo Ginzburg a montré qu'entre 1870 et 1880 se développa un nouveau procédé épistémologique dont les racines historiques remontent aux techniques de la lecture des traces dans le contexte de la chasse et de l'art de la divination ${ }^{45}$. Suivant son orientation casuistique sur le cas particulier et local, ce « paradigme de l'indice » incarne une sorte de contre-projet au type "galiléen » des sciences (naturelles), lesquelles sont basées sur l'abstraction et la généralisation. La psychanalyse, l'histoire de l'art et le roman policier forment pour Ginzburg les trois pôles du paradigme de l'indice. Ainsi Freud interprète-t-il les propos de ses patients comme des symptômes, comme des traces inconscientes de traumatismes passés, justifiant la psychanalyse. De la même manière, l'historien de l'art Giovanni Morelli parvient à identifier à l'aide de détails picturaux mineurs, dont les artistes n'ont généralement pas conscience, «la signature individuelle» des peintres avec une telle précision qu'il parvient à découvrir des cas spectaculaires de falsifications d'œuvres d'art. Enfin, c'est ainsi que le personnage littéraire Sherlock Holmes reconstruit avec une surprenante intuition et l'instinct d'un renard le déroulement d'un crime à partir de minuscules indices laissés involontairement. 
Aujourd'hui encore, l'efficacité du paradigme de l'indice demeure incontestée. Ginzburg lui-même, le fondateur de la «micro histoire $»^{46}$, a mis au jour à partir des protocoles de l'Inquisition les traces du savoir non écrit de la culture populaire rurale et l'a explicité sous la forme de narrations ${ }^{47}$. L'archéologie s'est définie elle-même comme " un paradigme de l'indice à l'œuvre ${ }^{48}$ en reconstituant à partir de traces, souvent laconiques et de second ordre, la structure d'un événement du passé. De cette façon, elle a également montré qu'elle était proche de la criminalistique: "la recherche, le relevé et l'interprétation de traces sont les points communs de l'archéologie et de la criminalistique $»^{49}$.

\section{Visualisation scientifique}

Bien que Ginzburg ait conçu le paradigme de l'indice comme une alternative au modèle "galiléen» de la formation de théories, propre aux sciences naturelles, le tournant praxéologique des science studies permet depuis trente ans d'aborder le travail des chercheurs en sciences naturelles à partir d'une nouvelle perspective ${ }^{50}$. Dans de nombreuses micro-études portant sur les recherches quotidiennes effectuées en laboratoire, on voit s'esquisser une "science dans l'action" qui, par son caractère interventionniste et sa tendance à mettre en scène ses objets, rappelle le fonctionnement d'un moteur de recherches, qui travaille souvent de manière fragmentaire et qui est destiné à produire des traces d'objets la plupart du temps invisibles. Ainsi, dans la technique expérimentale du marquage radioisotopique, des traces se forment. Cependant, leur particularité est qu'elles se désagrègent au moment où elles sont produites, rendant ainsi visible quelque chose qui n'existe (déjà) plus. Dans le contexte du relevé de traces tel qu'il est pratiqué au sein des sciences naturelles, la visualisation scientifique, c'est-à-dire la représentation graphique de données de mesure, de résultats de simulation et de suppositions théoriques, joue non plus seulement un rôle d'illustration, mais aussi de construction de la connaissance ${ }^{51}$. Les visualisations scientifiques ne donnent précisément pas à voir la représentation de leurs objets, car les données primaires de ces objets ne résultent souvent pas d'enregistrements optiques (il n'y a par exemple aucun éclairage sur ce qu'enregistre la microscopie à balayage électronique). Elles résultent plutôt d'informations cinétiques (" tactiles») et métriques, qui ne sont transformées en images qu'en dernier lieu ${ }^{52}$. Les images scientifiques ne montrent pas des objets préétablis, mais des suppositions ayant trait à ces objets, suppositions qui sont à la fois reconstituées à partir de traces formées expérimentalement et condensées de manière graphique.

\section{BIBLIOGRAPHIE}

Bernasconi, R. (1988) : « The Trace of Lévinas in Derrida », in : Bernasconi, R. / Wood, D. (éd.) :

Derrida and Différance, Evanston/ILL., p. 13-29. 
Cassirer, E. (1972) : La philosophie des formes symboliques, 3 tomes, trad. par O. Hansen Love, J. Lacoste et Cl. Fronty, Paris : Éditions de Minuit.

Daston, L. (2004) : Things that talk. Object Lessons from Art and Science, New York : Zone Books.

Derrida, J. (1967) : De la grammatologie, Paris : Les Éditions de Minuit.

Derrida, J. (1993) : «Signature - événement - contexte », in : id. : Marges de la philosophie, Paris : Les Éditions de Minuit, p. 365 sq.

Didi-Huberman, G. (2008) : La ressemblance par contact. Archéologie, anachronisme et modernité de l'empreinte, Paris : Les Éditions de Minuit.

Duden (1981) : Dasgroße Wörterbuch der deutschen Sprache in sechs Bänden, vol. 6, Mannheim et al. : Bibliographisches Institut.

Freud, S. (2010) : « Note sur le 'bloc-notes magiques' », in : id. : Huit études sur la mémoire et ses troubles, trad. de l'allemand par D. Messier, Paris : Gallimard.

Gawoll, H.-J. (1986/87) : « Spur : Gedächtnis und Andersheit. Teil I : Geschichte des Aufbewahrens ", Archiv für Begriffsgeschichte, p. 44-69.

Gawoll, H.-J. (1989) : « Spur : Gedächtnis und Andersheit. Teil II : Das Sein und die Differenzen Heidegger, Lévinas und Derrida », Archiv für Begriffsgeschichte, p. 269-296.

Ginzburg, C. (1980) : Le fromage et les vers. L'univers d'un meunier du XVIe siècle, trad. de l'italien par M. Aymard, Paris : Flammarion.

Ginzburg, C. (1984) : Les Batailles noctures. Sorcellerie et rituels agraires aux XVIe et XVIIe siècles, trad. de l'italien par G. Charuty, Paris : Flammarion.

Ginzburg, C. (1995) : « Spurensicherung. Der Jäger entziffert die Fährte, Sherlock Holmes nimmt die Lupe, Freud liest Morelli - die Wissenschaft auf der Suche nach sich selbst », in : id. : Spurensicherung. Die Wissenschaft auf der Suche nach sich selbst, Berlin : Wagenbach, p. 7-44.

Gomulicki, B. (1953) : The Development and Present Status of the Trace Theory of Memory, Cambridge : University Press.

Grimm, J. / Grimm, W. (1854-1860) : Deutsches Wörterbuch, Leipzig : S. Hirzel.

Heidegger, M. (1988) : Les Hymnes de Hölderlin «La Germanie » et « Le Rhin », trad. de l'allemand par F. Fédier et J. Hervier, "Paris : Gallimard.

Heider, F. (1927) : « Ding und Medium », Symposion 1, p. 109-157.

Heintz, B. / Huber, J. (éd.) (2001) : Mit dem Auge denken. Strategien der Sichtbarmachung in wissenschaftlichen und virtuellen Welten, Zurich : Voldemeer.

Hennig, J. (2006) : « Die Versinnlichung des Unzugänglichen - Oberflächendarstellungen in der zeitgenössischen Mikroskopie », in : Heßler, M. : Konstruierte Sichtbarkeiten. Wissenschafts- und Technikbilder seit der Frühen Neuzeit, Munich : W. Fink, p. 99-116.

James, W. (1890) : Principles of Psychologie, New York : Dover Publication Inc.

Krämer, S. / Kogge, W. / Grube, G. (éd.) : Spur : Spurenlesen als Orientierungstechnik und Wissenskunst, Francfort-sur-le-Main : Suhrkamp, 2007.

Kümmel, Ch. (2003) : « Wie weit trägt ein Indizienbeweis ? Zur archäologischen Überführung von Grabräubern ", in : Veit, Th. et al.: Spuren und Botschaften. Interpretationen materieller Kultur, Münster : Waxmann, p. 135-156.

Leibniz, G. W. (1991) : La monadologie, éd. critique établie par É. Boutroux, Paris : Le livre de Poche. 
Lenoir, Th. (éd.) (1998) : Inscribing Science. Scientific Texts ans the Materiality of Communication, Stanford/Ca, Stanford University Press.

Lévinas, E. (1988a) : « Enigme et phénomène ", in : id. : En découvrant l'existence avec Husserl et Heidegger, Paris : Librairie Philosophique J. Vrin, p. 203-216.

Lévinas, E. (1988b) : « La trace de l'autre », in : id. : En découvrant l'existence avec Husserl et Heidegger, Paris : Librairie Philosophique J. Vrin., p. 187-202.

Mante, G. (2003) : «Spuren lesen. Die Relevanz kriminalistischer Methoden für die archeologische Wissenschaft ", in : Veit, Th. et al.: Spuren und Botschaften. Interpretationen materieller Kultur, Münster : Waxmann, p. 157-172.

Metken, G. (1977) : Spurensicherung. Kunst als Anthropologie und Selbsterforschung. Fiktive Wisssenschaften in der heutigen Kunst, Cologne : DuMont.

Metken, G. (1996) : Spurensicherung. Eine Revision. Texte 1977-1995, Dresde : Philo Verlagsgesellschaft.

Peirce, Ch. S. (2001) : Euvres philosophiques, vol. 1 : Pragmatisme et Pragmaticisme, trad. de l'anglais (États-Unis) par Cl. Tiercelin et P. Thibaud, Paris : Les Éditions du Cerf.

Platon : Théétète, trad. par M. Narcy, Paris : Flammarion, 1994.

Pöggeler, O. (1992) : Neue Wege mit Heidegger, Fribourg-en-Brisgau : K. Adler.

Reichertz, J. (1998) : « Von Haaren und Nägeln. Zur impliziten Anthropologie von Ch. S. Peirce », Kodidas/Code 3-4, p. 287-304.

Saussure, F. de (1972) : Cours de linguistique générale, éd. par Ch. Bally et A. Séchehaye, Paris : Éditions Payot.

Sebeok, T. A. / Umiker-Sebeok, J. (1982) : «Du kennst meine Methode.» Charles S. Peirce und Sherlock Holmes, Francfort-sur-le-Main : Suhrkamp.

Spitznagel, A. (2001) : « Auf der Spur der Spuren », in : Arburg, H.-G. von / Gamper, M. / Stadler, U. (éd.) : «Wunderliche Figuren ». Über die Lesbarkeit von Chiffrenschriften, Munich : Wilhelm Fink Verlag, p. 239-259.

Stegmaier, W. (1996) : « Die Zeit und die Schrift. Berührungen zwischen Lévinas und Derrida », Allgemeine Zeitschrift für Philosophie,1, p. 3-24.

Vattimo, G. (1996) : « La trace de la trace », in : Derrida, J. / Vattimo, G. (éd.) : La religion. Séminaire de Capri, Paris : Éd. du Seuil, p. 105-122.

Wirth, U. (1995) : « Abduktion und ihre Anwendung. Ein Forschungsbericht », Zeitschrift für Semiotik, 17, p. 405-424.

\section{NOTES}

1. NDLR : Ce texte est un extrait de l'introduction à l'ouvrage Krämer / Kogge / Grube (2007).

2. Saussure (1972), p. 158 sqq.

3. Cf. Cassirer (1972).

4. Au sujet de la réhabilitation des choses cf. Daston (2004).

5. Peter Geimer montre dans le présent volume à quel point il est difficile, dans le cas de traces, de délimiter l'acte de représenter de celui de présenter. Il s'appuie sur l'exemple de la photographie conçue comme une trace du réel. 
6. Avant que « ressentir» [ «Spüren »] n'ait le sens d'« éprouver» et d'« apercevoir », ce verbe signifie «relever une piste»; Grimm, J./ Grimm, W. (1854-1860), entrée «Spüren », t. 17, col. 243.

7. Grimm, J. / Grimm, W. (1854-1860), entrée « Spur », t. 17, col. 239.

8. Duden (1981), entrée « Spur », p. 2464.

9. "Adhérence il y a eu, mais adhérence à qui, à quoi, à quel instant, à quel corps-origine ?» demande Georges Didi-Huberman dans sa description du paradoxe de l'empreinte. (DidiHuberman [2008], p. 309).

10. Lévinas (1988a), p. 208.

11. Cf. à ce sujet Werner Stegmaier dans le présent volume.

12. Helmut Pape renvoie expressément à cet aspect dans ce volume.

13. Lévinas (1988b), p. 200.

14. Werner Kogge met en évidence, dans ce volume, l'implication qu'exige toute lecture de traces.

15. Cf. Wolfram Hogrebe dans le présent volume.

16. Heider (1927), p. 115 sqq.

17. Gernot Grube présente dans ce volume la méthode de la sélection de traces.

18. Ceci fait référence à l'acception technique que l'on réserve à la trace en tant que partie d'une surface (comme par exemple dans le cas d'une voie de circulation).

19. Dans sa contribution, Jo Reichertz dote cette créativité d'une telle valeur, qu'il ne parle plus de lecture, mais de « construction de traces" (dans cet ouvrage).

20. Uwe Wirth renvoie dans le présent volume à de telles interférences.

21. Font entre autres partie de ce cercle les artistes suivants : Nikolaus Lang, Christian Boltanski, Anne et Patrick Poirier et Jochen Gerz.

22. Metken (1977) et Metken (1996).

23. Mirjam Schaub démontre dans ce volume que cela vaut aussi pour d'autres courants artistiques qui mettent en scène et impriment des traces.

24. Spitznagel (2001), p. 259.

25. Sybille Krämer accentue dans ce volume le rôle que joue l'interprétation de la trace en la rapprochant d'une bifurcation entre, d'une part, le paradigme indiciaire en tant qu'art scientifique positif et, d'autre part, une métaphysique de la trace en tant qu'expérience de la limite et du renoncement.

26. Ginzburg (1995), p. 15.

27. Cf. Wolfram Hogrebe dans ce volume, p. 289.

28. Dans le sens où l'on en conclut, à partir d'un résultat, à la spécification d'une hypothèse explicative en se basant sur une « phrase générale».

29. Cf. Peirce (2001), p. 431 sqq.

30. Uwe Wirth place l'abduction chez Peirce entre la psychologie et la logique ; cf. Wirth (1995), p. 408.

31. Thomas A. Sebeok et Jean Umiker-Sebeok attirent avant tout l'attention sur ce point dans Sebeok / Umiker-Sebeok (1982) p. 38. Cf. aussi Reichertz (1998), p. 297.

32. À ce propos, on se rapportera au concept psychologique de la "sagacity », - la perspicacité, développé par William James ; cf. James (1890), cité d'après Spitznagel (2001), p. 259.

33. Bronislaw Gomulicki donne un bon aperçu de la théorie de la trace dans le contexte de la mémoire; cf. Gomulicki (1953). Sur la tradition philosophique de l'engramme, cf. Gawoll (1986/87).

34. Cf. Platon : Théétète 191d (trad. fr, p. 250 sq).

35. Cf. Leibniz (1991), p. 143.

36. Cf. Leibniz (1991), 129sq ; 137 sq.

37. Gawoll (1986/87), entrée « Spur » [trace], p. 52. 
38. Cf. Freud (2010), p. 135 sqq.

39. Sur Plotin cf. Gawoll (1986/87), p. 1550 sqq.

40. Cité d'après Gawoll (1986/87), p. 1550 sqq.

41. Cf. aussi Vattimo (1996).

42. Cf. Heidegger (1988), p. 40 sqq. Sur Heidegger : cf. Gawoll (1989) et Pöggeler (1992).

43. Lévinas (1988b).

44. Derrida (1967) et (1993). Pour une étude du concept de trace chez Lévinas et Derrida, cf. Bernasconi (1988); Gawoll (1989); Stegmaier (1996).

45. Cf. Ginzburg (1995), p. 15.

46. L'historien Giovanni Levi en est le co-fondateur.

47. Cf. Ginzburg (1980), (1984).

48. Cf. Kümmel (2003), p. 143.

49. Mante (2003) p. 157 sqq.

50. On citera à titre d'exemple Lenoir (1998).

51. À ce sujet, cf. Heintz / Huber (2001).

52. Cf. Hennig (2006).

INDEX

Mots-clés : trace, lecture, lecture de traces

Schlüsselwörter : Spur, Lesen, Spurenlesen

\section{AUTEURS}

SYBILLE KRÄMER

Sybille Krämer, professeur de philosophie à la FU Berlin. Pour plus d'informations, voir la notice suivante. 